\title{
EFICÁCIA DE DADOS HYPERION/EO-1 PARA IDENTIFICAÇÃO DE ALVOS AGRÍCOLAS: COMPARAÇÃO COM DADOS ETM ${ }^{+} / L A N D S A T-7$
}

\author{
DANIELA A. TISOT ${ }^{1}$, ANTONIO R. FORMAGGIO ${ }^{2}$, CAMILO D. RENNÓ ${ }^{3}$, \\ LÊENIO S. GALVÃ ${ }^{2}$
}

\begin{abstract}
RESUMO: O objetivo desta pesquisa foi avaliar o uso de dados hiperespectrais Hyperion/EO-1 na discriminação de alvos agrícolas, comparando a acurácia de classificação obtida pelos dados desse sensor à obtida por dados multiespectrais $\mathrm{ETM}^{+} /$Landsat-7. Para isso, alvos agrícolas da região de Franca - SP, com diferenças espectrais bem definidas e outros alvos com diferenças espectrais sutis, imageados por ambos os sensores, em 16 de julho de 2002, foram discriminados utilizando o classificador supervisionado de Máxima Verossimilhança (MaxVer). Os alvos agrícolas com diferenças espectrais bem definidas foram caracterizados por seis classes de uso e cobertura do solo; já os com diferenças espectrais sutis, por cinco classes de variedades de cana-de-açúcar. Quando os dados $\mathrm{ETM}^{+}$foram classificados, a acurácia foi de 91,5\% para as classes de uso e cobertura do solo e de 67,6\% para as classes de variedades de cana-de-açúcar, enquanto, para os dados do sensor Hyperion, a acurácia de classificação foi de $94,9 \%$ e de $87,1 \%$, respectivamente, demonstrando, assim, a importância do uso de dados hiperespectrais na discriminação de alvos agrícolas com características espectrais semelhantes.
\end{abstract}

PALAVRAS-CHAVE: classificação digital, dados hiperespectrais, dados multiespectrais.

\section{HYPERION/EO-1 HYPERSPECTRAL DATA EFFICACY FOR AGRICULTURAL TARGETS IDENTIFICATION: A COMPARISON WITH ETM ${ }^{+} / L A N D S A T-7$ MULTISPECTRAL DATA}

\begin{abstract}
The aim of this work was to assess Hyperion/EO-1 hyperspectral data to discriminate agricultural fields, comparing that data classification accuracy with $\mathrm{ETM}^{+} /$Landsat-7 multi-spectral data classification accuracy. For this purpose, agricultural targets with well-defined and subtle spectral differences in Franca municipality, São Paulo State, Brazil, imaged by both sensors in July, 2002, were discriminated by using a supervised classification (Maximum Likelihood) algorithm. Agricultural targets with well-defined spectral differences were characterized by six land use/land cover classes, while five sugarcane varieties were used as targets with subtle spectral differences. When the broadband $\mathrm{ETM}^{+}$data were classified, the overall accuracy was $91.5 \%$ for six land use/land cover classes and $67.6 \%$ for five sugarcane variety classes, while for narrowband Hyperion data the accuracies were $94.9 \%$ and $87.1 \%$, respectively. This result shows the importance of hyperspectral data use for agricultural targets discrimination with similar spectral characteristics.
\end{abstract}

KEYWORDS: digital classification, hyperspectral data, multispectral data.

\footnotetext{
${ }^{1}$ Eng ${ }^{\mathrm{a}}$ Agrônoma, Doutoranda do Curso de Pós-Graduação em Sensoriamento Remoto, Instituto Nacional de Pesquisas Espaciais (INPE/MCT), São José dos Campos - SP, Fone: (0XX12) 3945.6458, dtisot@dsr.inpe.br

${ }^{2}$ Pesquisador Titular, Divisão de Sensoriamento Remoto (DRS), Instituto Nacional de Pesquisas Espaciais (INPE/MCT), São José dos Campos - SP.

${ }^{3}$ Pesquisador Doutor, Divisão de Processamento de Imagens (DPI), Instituto Nacional de Pesquisas Espaciais (INPE/MCT), São José dos Campos - SP.

Recebido pelo Conselho Editorial em: 4-9-2006

Aprovado pelo Conselho Editorial em: 30-3-2007
} 


\section{INTRODUÇÃO}

A produção de mapas temáticos, como os de uso e cobertura do solo por meio da classificação de imagens, é uma das aplicações mais utilizadas do sensoriamento remoto, sendo o aumento da acurácia de classificação um dos principais enfoques de estudo.

O classificador supervisionado de Máxima Verossimilhança (MaxVer) é um método paramétrico, o qual assume que cada classe pode ser modelada por uma distribuição gaussiana multivariada. A eficácia do algoritmo MaxVer depende principalmente do número de pixels incluídos nas amostras de treinamento usadas para estimar os parâmetros de distribuição, que são o vetor média e a matriz de co-variância. Esse algoritmo deve ser aplicado quando o analista conhece bem a imagem a ser classificada, para que possa definir classes que sejam representativas. Detalhes sobre esse classificador podem ser encontrados em SWAIN \& DAVIS (1978) e em RICHARDS \& JIA (1999).

Para algumas aplicações, como a classificação de alvos agrícolas, as imagens geradas a partir de sensores de bandas espectrais estreitas podem melhorar a capacidade de discriminação e a acurácia de classificação, comparadas às imagens geradas a partir de sensores de bandas espectrais largas.

Recentes estudos indicam as vantagens do uso de dados obtidos a partir de bandas estreitas, localizadas em específicas posições do espectro, na obtenção de informações qualitativas ou quantitativas da vegetação, porém em poucos deles foram utilizados dados hiperespectrais oriundos de sensores orbitais para o estudo de alvos agrícolas. Dentre os estudos realizados com dados do sensor Hyperion, destacam-se: detecção de doença foliar em cana-de-açúcar por meio de índices hiperespectrais de vegetação (APAN et al., 2004); discriminação de variedades de cana-de-açúcar usando valores de reflectância das bandas individuais, razões de reflectância e índices hiperespectrais (GALVÃO et al., 2005), e comparação da classificação de florestas obtida a partir de dados de sensores multiespectrais, como a obtida a partir de dados do sensor Hyperion (GOODENOUGH et al., 2003; THENKABAIL et al., 2004).

Assim, o objetivo da presente pesquisa foi avaliar o uso de dados hiperespectrais Hyperion/EO-1 na discriminação de alvos agrícolas. Para isso, a acurácia de classificação obtida pelos dados desse sensor foi comparada à obtida por dados multiespectrais $\mathrm{ETM}^{+} /$Landsat-7, tanto para um grupo de alvos agrícolas com diferenças espectrais bem definidas quanto para outro grupo com diferenças espectrais sutis.

\section{MATERIAL E MÉTODOS}

O estudo foi realizado em área localizada na região agrícola de Franca - SP (2045'46.64”'S e $47^{\circ} 26^{\prime} 24.96^{\prime \prime}$ W) (Figura 1), onde predominam, além das pastagens, as culturas da cana-de-açúcar e do café, cultivados em latossolos e em relevo suave ondulado. Utilizou-se de uma imagem $\mathrm{ETM}^{+} /$Landsat-7 e de uma Hyperion/EO-1, ambas adquiridas em 16 de julho de 2002, com defasagem temporal de apenas um minuto entre as passagens dos dois respectivos satélites.

O instrumento Hyperion está a bordo do satélite Earth Observing-1 (EO-1), da NASA, sendo esse sensor hiperespectral capaz de imagear a superfície terrestre em 220 bandas espectrais entre 400 e $2.500 \mathrm{~nm}$, conta com resolução espacial de $30 \mathrm{~m}$ e resolução espectral nominal de $10 \mathrm{~nm}$, mapeando faixa de $7,5 \mathrm{~km}$ x $100 \mathrm{~km}$ em cada cena, operando no modo pushbroom com alta confiabilidade radiométrica (PEARLMAN et al., 2003; NASA, 2007).

Após a conversão dos dados para valores de reflectância de superfície usando o "software" Atmospheric Correction Now (ACORN) (IMSPEC, 2001) para a imagem Hyperion e o método descrito por GURTLER et al. (2005) para a imagem ETM+, as imagens foram registradas tendo como base uma carta topográfica do município de Franca (escala 1:250.000).

As bandas em torno das faixas de absorção atmosférica, posicionadas ao redor de $1.400 \mathrm{~nm}$ e de $1.900 \mathrm{~nm}$, foram excluídas da análise dos dados Hyperion. No intuito de reduzir a redundância 
de informações das 151 bandas restantes e o tempo de processamento dos dados da imagem hiperespectral, foi realizada a Análise por Componentes Principais (ACP).

Utilizando o método de classificação supervisionada "pixel-a-pixel" (MaxVer), mapas de classificação foram gerados a partir das seis bandas refletivas do sensor $\mathrm{ETM}^{+}$e da ACP do sensor Hyperion. Os critérios adotados para a seleção das componentes principais (CPs) utilizadas foram a análise dos autovalores e a qualidade visual das imagens dessas componentes.

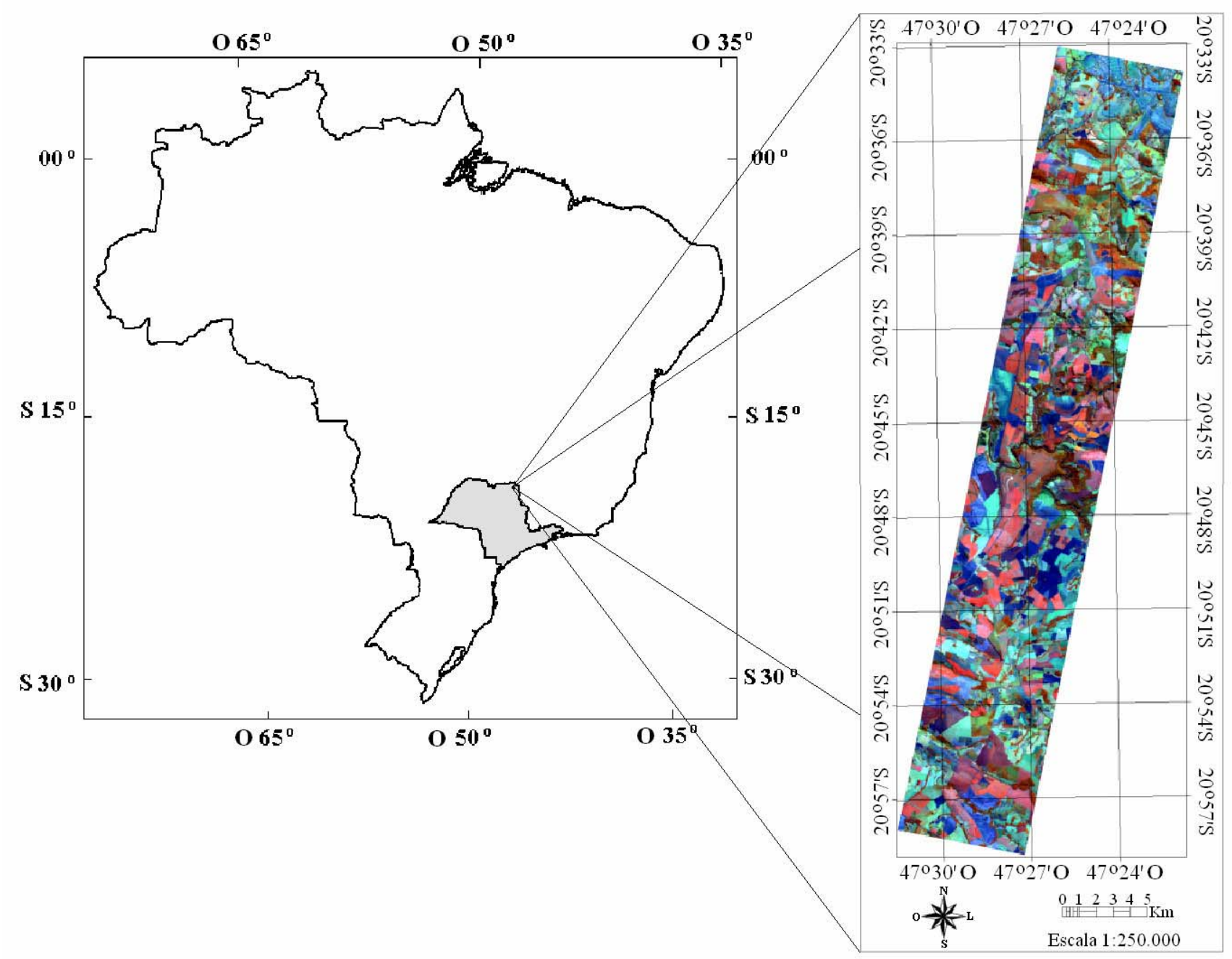

FIGURA 1. Localização da área de estudo, no município de Franca - SP, e imagem Hyperion/EO-1 utilizada.

Dados de campo previamente coletados foram utilizados na caracterização das classes de estudo. Usando o "software" ENVI (SULSOFT, 2007), amostras foram selecionadas aleatoriamente nas áreas das classes de interesse, tomando-se o cuidado de tomar amostras de mesmos tamanhos e nos mesmos locais, para ambos os conjuntos de dados (ETM+ e Hyperion), a fim de propiciar as melhores condições comparativas possíveis.

É oportuno indicar que os métodos de classificação podem ser de duas categorias: a classificação supervisionada e a não-supervisionada. Na segunda, não há qualquer conhecimento prévio do classificador sobre os atributos das classes estudadas na cena, enquanto, na classificação supervisionada, o classificador orienta sua busca de classes a partir de amostras de treinamento feitas a priori com as classes de interesse presentes na cena. Para maiores detalhamentos sobre classificadores e amostragens, recomenda-se SULSOFT (2007).

A fim de avaliar e de comparar a classificação dos dados multiespectrais $\left(\mathrm{ETM}^{+}\right)$com a dos dados hiperespectrais (Hyperion), dois enfoques foram dados neste trabalho: classificação de classes de uso e cobertura do solo presentes na região, predominando diferenças espectrais 
marcantes entre as quais a classificação de subclasses dentro de uma mesma classe, no intuito de discriminar diferenças espectrais mais sutis entre elas. No primeiro caso, as classes escolhidas foram: cana-de-açúcar, café, pastagem, palha, solo exposto e mata. No segundo, apenas a classe cana-de-açúcar foi estudada, subdividindo-a em subclasses de variedades: RB72-454, SP80-1816, SP80-1842, SP81-3250 e SP87-365, uma vez que todos os talhões da classe cana-de-açúcar estudados estavam em condições de cobertura completa da superfície.

Para comparar os resultados obtidos entre as classificações de ambos os sensores, a matriz de confusão foi gerada comparando cada classificação com o mapa de referência, sendo o Coeficiente Kappa calculado de acordo com HUDSON \& RAMM (1987) e CONGALTON \& GREEN (1999).

\section{RESULTADOS E DISCUSSÃO}

A partir da análise das 151 PCs Hyperion obtidas, foram selecionadas as oito primeiras componentes correspondentes a aproximadamente $100 \%$ da variância dos dados dessa imagem, o que indica grande quantidade de informação redundante adquirida pelo sensor nessa imagem (Figura 2). Apesar de a oitava componente ter contribuído com apenas 0,042\% da variância dos dados, essa foi selecionada devido à possibilidade de identificação visual dos alvos da imagem nessa componente.

Componentes Principais Hyperion

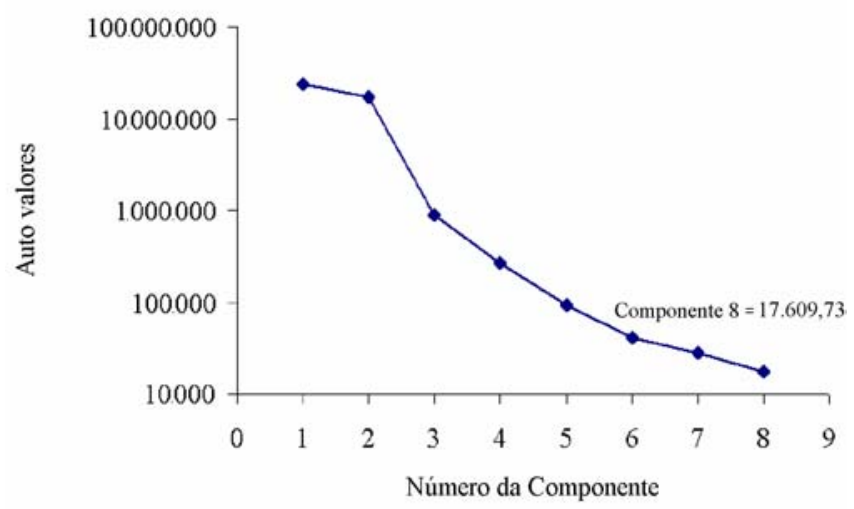

FIGURA 2. Autovalores (escala logaritmica) dos dados Hyperion. Da componente 9 à 151, valores inferiores a 0,00000 .

\section{Discriminação de alvos agrícolas com diferenças espectrais marcantes}

Utilizando os dados das seis bandas refletivas do sensor $\mathrm{ETM}^{+}$, a acurácia para discriminar as seis grandes classes de uso e cobertura do solo (mata, pastagem, palha, solo exposto, café e canade-açúcar) da cena agrícola estudada foi de $91,5 \%$, e o valor do Kappa, igual a 0,88. A classificação Hyperion que mais se aproximou da $\mathrm{ETM}^{+}$foi a correspondente às três primeiras CPs, com acurácia de 89,7\% (Kappa =0,85). Ambas as classificações diferiram significativamente a $1 \%$.

No que diz respeito à discriminação das classes de uso e cobertura do solo utilizando as oito primeiras CPs Hyperion, a acurácia de classificação foi de 94,9\%, e o valor de Kappa, igual a 0,93, tendo-se verificado que as bandas nas regiões espectrais do vermelho e do infravermelho próximo e médio foram as que mais contribuíram e mais se correlacionaram com essas CPs, o que está concordante com o que foi encontrado por GALVÃO et al. (2005).

Na classificação dos dados multiespectrais (Tabela 1), os menores erros de comissão (pixels de outras classes que foram atribuídos à classe de referência) e omissão (pixels pertencentes a uma classe de referência que foram atribuídos a outras classes) foram observados para a classe cana-deaçúcar, com $1,76 \%$ e $2,84 \%$, respectivamente, conferindo a essa o melhor desempenho do classificador. 
TABELA 1. Matriz de confusão para as classes de uso e cobertura do solo geradas pelo MaxVer a partir das seis bandas refletivas $\mathrm{ETM}^{+}$.

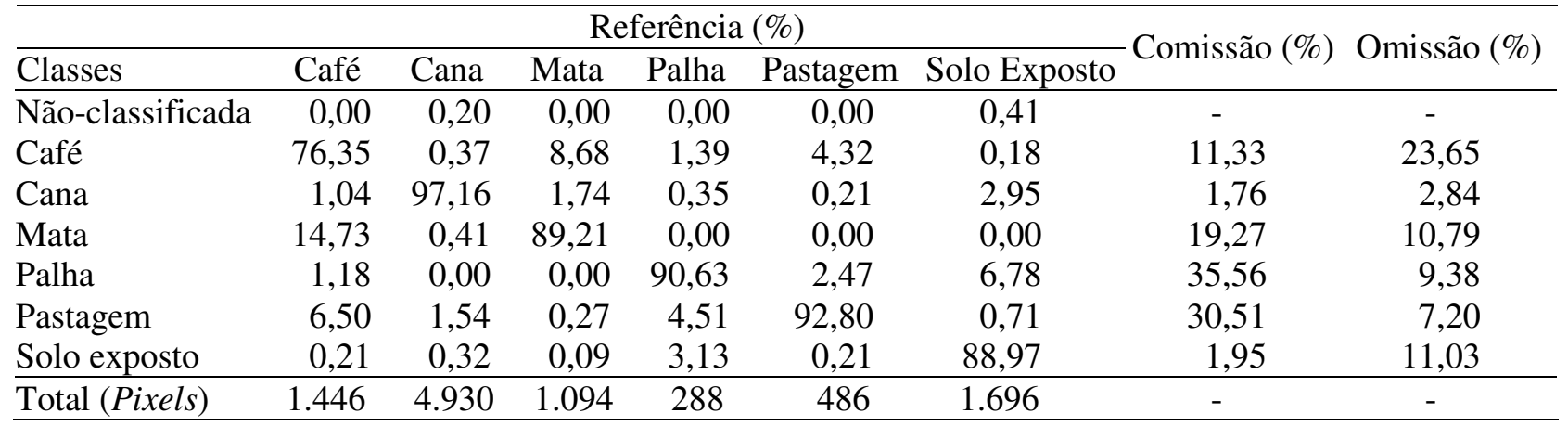

Apesar de 90,63\% dos pixels pertencentes à classe palha e de 92,80\% dos pertencentes à classe pastagem terem sidos classificados corretamente, os maiores erros de comissão foram observados para essas classes. Isso indica que 35,56\% dos pixels que foram classificados como palha e $30,51 \%$ dos que foram classificados como pastagem, pertencem, na realidade, a outras classes. Provavelmente, isso deve ter ocorrido em função de as imagens utilizadas terem sido da época seca do ano (julho) e, dessa forma, várias áreas de pastagens encontravam-se com respostas espectrais semelhantes às de palha.

Na classificação dos dados hiperespectrais (Tabela 2), a melhor classificação também foi encontrada para a classe cana-de-açúcar, porém com menores erros de comissão e omissão $(0,73 \%$ e $1,18 \%$, respectivamente).

TABELA 2. Matriz de confusão para as classes de uso e cobertura do solo geradas pelo MaxVer a partir das oito primeiras CPs Hyperion.

\begin{tabular}{|c|c|c|c|c|c|c|c|c|}
\hline & \multicolumn{6}{|c|}{ Referência $(\%)$} & \multirow{2}{*}{ Comissão (\%) } & \multirow{2}{*}{ Omissão (\%) } \\
\hline Classes & Café & Cana & Mata & Palha & Pastagem & Solo Exposto & & \\
\hline Não-classificada & 0,00 & 0,20 & 0,00 & 0,00 & 0,00 & 0,41 & - & - \\
\hline Café & 87,00 & 0,14 & 9,14 & 1,74 & 4,12 & 0,12 & 9,63 & 13,00 \\
\hline Cana & 0,21 & 98,82 & 0,46 & 0,00 & 0,41 & 1,53 & 0,73 & 1,18 \\
\hline Mata & 10,51 & 0,10 & 90,40 & 0,00 & 0,00 & 0,00 & 13,70 & 9,60 \\
\hline Palha & 1,18 & 0,00 & 0,00 & 92,01 & 0,41 & 4,01 & 24,72 & 7,99 \\
\hline Pastagem & 1,04 & 0,57 & 0,00 & 0,69 & 95,06 & 0,06 & 9,06 & 4,94 \\
\hline Solo exposto & 0,07 & 0,16 & 0,00 & 5,56 & 0,00 & 93,87 & 1,55 & 6,13 \\
\hline Total (Pixels) & 1.446 & 4.930 & 1.094 & 288 & 486 & 1.696 & - & - \\
\hline
\end{tabular}

Em ambas as classificações, as classes mais confundidas entre si foram café e mata, uma vez que há grande similaridade espectral entre ambas.

Apesar de a acurácia de classificação Hyperion, utilizando oito CPs, ter apresentado resultado estatisticamente superior à $\mathrm{ETM}^{+}$e os erros de comissão e omissão da primeira terem sido menores para todas as classes estudadas, pode-se dizer que a qualidade das duas classificações foi visualmente semelhante (Figura 3). Embora o sensor $\mathrm{ETM}^{+}$colete volume de dados aproximadamente 75 vezes menor quando comparado ao sensor Hyperion, o posicionamento e a largura das bandas espectrais são suficientes para classificar alvos agrícolas com diferenças espectrais predominantemente grosseiras. 


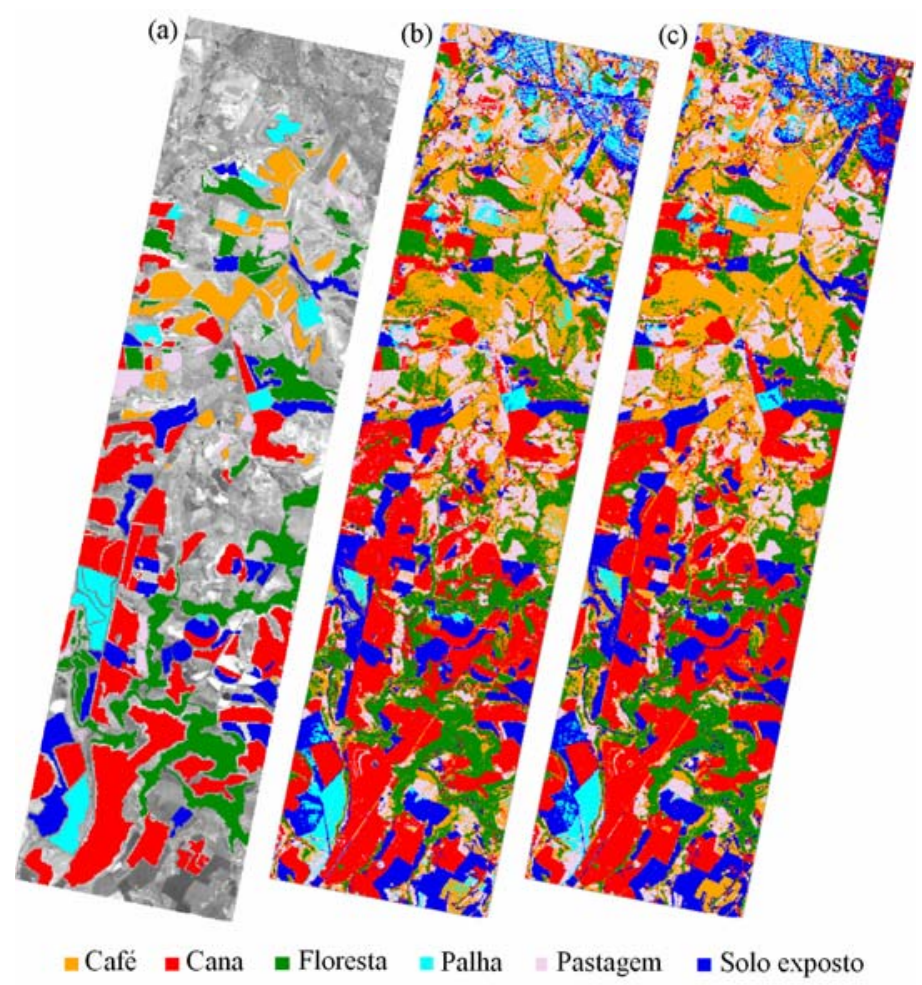

FIGURA 3. Composição colorida falsa-cor com as bandas do sensor Hyperion centradas em $833 \mathrm{~nm}(\mathrm{R}), 1.659 \mathrm{~nm}(\mathrm{G})$ e $660 \mathrm{~nm}(\mathrm{~B})$, indicando as áreas utilizadas na classificação do uso e cobertura do solo (a), mapas temáticos de uso e de cobertura do solo gerados a partir da classificação das imagens das seis bandas refletivas do sensor $\mathrm{ETM}^{+}$(b) e das primeiras oito CPs do sensor Hyperion (c).

\section{Discriminação de alvos agrícolas com diferenças espectrais sutis}

Utilizando os dados das seis bandas refletivas do sensor $\mathrm{ETM}^{+}$, a acurácia para discriminar as cinco variedades de cana-de-açúcar (classes com diferenças espectrais sutis) foi de 67,6\%, equivalendo à classificação apenas das três primeiras CPs hiperespectrais, cuja acurácia foi de $67,7 \%$. Para ambas as classificações, o valor do Kappa foi de 0,58.

Quanto à classificação obtida com as oito primeiras CPs Hyperion, a acurácia foi de $87,1 \%$, isto é, $19,5 \%$ superior à obtida para a imagem $\mathrm{ETM}^{+}$, e com valor Kappa igual a 0,86.

$\mathrm{Na}$ classificação $\mathrm{ETM}^{+}$, os menores erros de omissão foram atribuídos à variedade SP87-365, conferindo a melhor classificação aos pixels pertencentes a essa classe (Tabela 3). Porém, o erro de comissão elevado indica que $40,35 \%$ dos pixels que foram classificados como SP87-365 pertencem, na realidade, a outras classes. Já para a variedade RB72-454, os erros foram os maiores, tanto de comissão quanto de omissão, sendo $58,14 \%$ dos pixels classificados para essa classe pertencem, na realidade, a outras classes de variedades, e 51,82\% dos pixels pertencentes à classe RB72-454 foram atribuídos às demais. Esse elevado valor de omissão colaborou, por conseqüência, com o erro de comissão de pixels das demais classes de variedades, principalmente da SP80-1816 e SP81-3250. Nesse sentido, é importante destacar, por exemplo, que a discriminação espectral da cultivar SP80-1842, em relação às outras cultivares, é influenciada por sua menor reflectância no infravermelho próximo, por suas mais profundas bandas de absorção, em $2.013 \mathrm{~nm}$ e em $2.304 \mathrm{~nm}$, além de bandas mais rasas, em $983 \mathrm{~nm}$ e em $1.205 \mathrm{~nm}$. Diferenças relacionadas com as estruturas dos dosséis produziram maiores reflectâncias para as cultivares mais planófilas (p.ex., SP81-3250) do que para as mais erectófilas (p.ex., SP80-1842), o que está de acordo com GALVÃO et al. (2005).

Na classificação Hyperion, o melhor resultado de classificação também foi atribuído à classe da variedade SP87-365, porém o erro de comissão foi 37,82\% inferior, e o de omissão, 11,17\% inferior aos gerados pela classificação $\mathrm{ETM}^{+}$(Tabela 4). 
TABELA 3. Matriz de confusão para as classes de variedades de cana-de-açúcar geradas pelo MaxVer a partir das seis bandas refletivas $\mathrm{ETM}^{+}$.

\begin{tabular}{lrrrrrrr}
\hline \multicolumn{9}{c}{ Referência (\%) } \\
\cline { 1 - 6 } Classes & RB72-454 & SP80-1816 & SP80-1842 & SP81-3250 & SP87-365 & \multirow{2}{*}{ Comissão (\%) Omissão (\%) } \\
\hline Não-classificada & 3,41 & 3,33 & 2,35 & 0,70 & 3,88 & - & - \\
RB72-454 & 48,18 & 15,15 & 6,86 & 22,18 & 1,94 & 58,14 & 51,82 \\
SP80-1816 & 27,74 & 69,96 & 13,14 & 10,56 & 10,68 & 22,58 & 30,04 \\
SP80-1842 & 7,54 & 1,93 & 74,31 & 0,35 & 0,97 & 12,87 & 25,69 \\
SP81-3250 & 10,46 & 2,63 & 0,20 & 63,38 & 0,00 & 29,13 & 36,62 \\
SP87-365 & 2,68 & 7,01 & 3,14 & 2,82 & 82,52 & 40,35 & 17,48 \\
\hline Total (Pixels) & 411 & 1.142 & 510 & 284 & 206 & - & - \\
\hline
\end{tabular}

TABELA 4. Matriz de confusão para as classes de variedades de cana-de-açúcar geradas pelo MaxVer a partir das oito primeiras CPs Hyperion.

\begin{tabular}{lrrrrrrr}
\hline & \multicolumn{9}{c}{ Referência (\%) } & \multicolumn{2}{c}{ Comissão (\%) Omissão (\%) } \\
\hline Classes & RB72-454 & SP80-1816 & SP80-1842 & SP81-3250 & SP87-365 & - \\
Não-classificada & 3,41 & 3,33 & 2,35 & 0,70 & 3,88 & - & 27,49 \\
RB72-454 & 72,51 & 5,95 & 1,96 & 0,70 & 0,00 & 21,16 & 11,56 \\
SP80-1816 & 18,49 & 88,44 & 4,51 & 6,69 & 2,43 & 10,86 & 9,22 \\
SP80-1842 & 1,95 & 1,14 & 90,78 & 0,00 & 0,00 & 4,34 & 8,45 \\
SP81-3250 & 3,41 & 0,96 & 0,20 & 91,55 & 0,00 & 9,09 & 6,31 \\
SP87-365 & 0,24 & 0,18 & 0,20 & 0,35 & 93,69 & 2,53 & - \\
\hline Total (Pixels) & 411 & 1.142 & 510 & 284 & 206 & - & - \\
\hline
\end{tabular}

Esses resultados comprovam o melhor desempenho das imagens hiperespectrais em relação às imagens multiespectrais na discriminação de alvos agrícolas com diferenças espectrais muito sutis, como discriminação de variedades de cana-de-açúcar (Figuras 4 e 5).

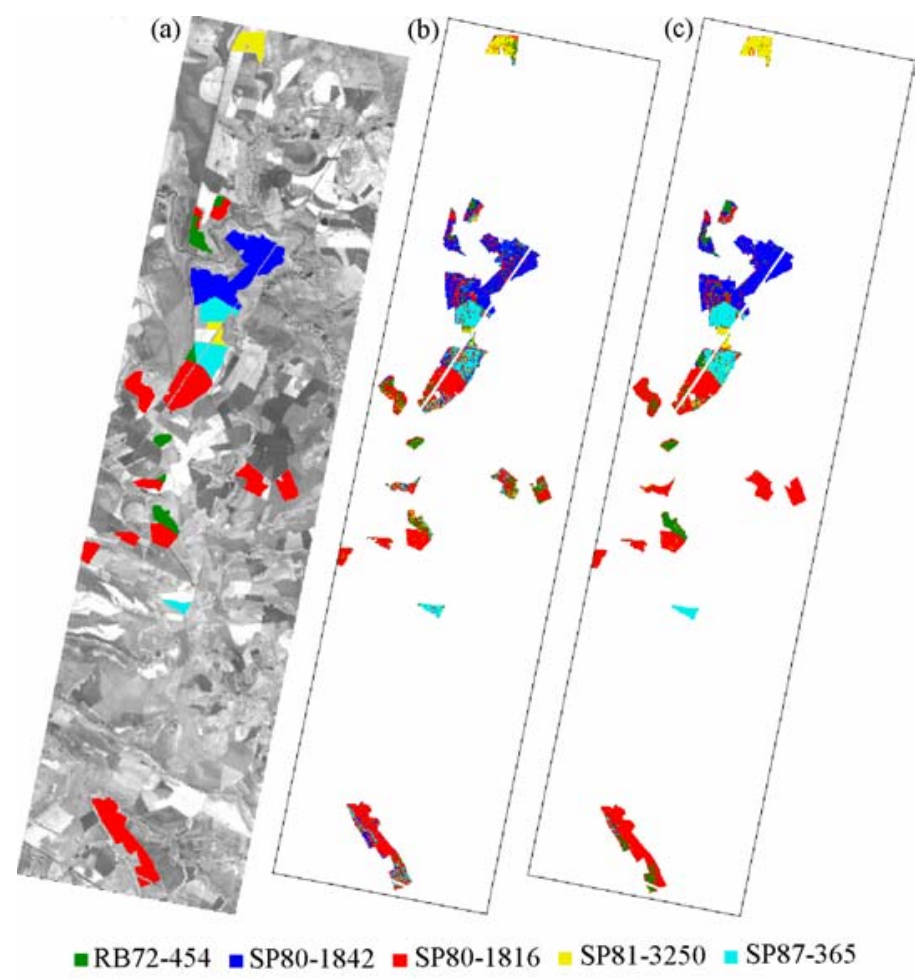

FIGURA 4. Composição colorida falsa-cor com as bandas do sensor Hyperion centradas em 833 $\mathrm{nm}(\mathrm{R}), 1.659 \mathrm{~nm}(\mathrm{G})$ e $660 \mathrm{~nm}(\mathrm{~B})$, indicando as áreas de cana-de-açúcar utilizadas na classificação das variedades (a); mapas temáticos de diferenciação de variedades de cana-de-açúcar gerados a partir da classificação das imagens das seis bandas refletivas do sensor $\mathrm{ETM}^{+}$(b), e das primeiras oito CPs do sensor Hyperion (c). 


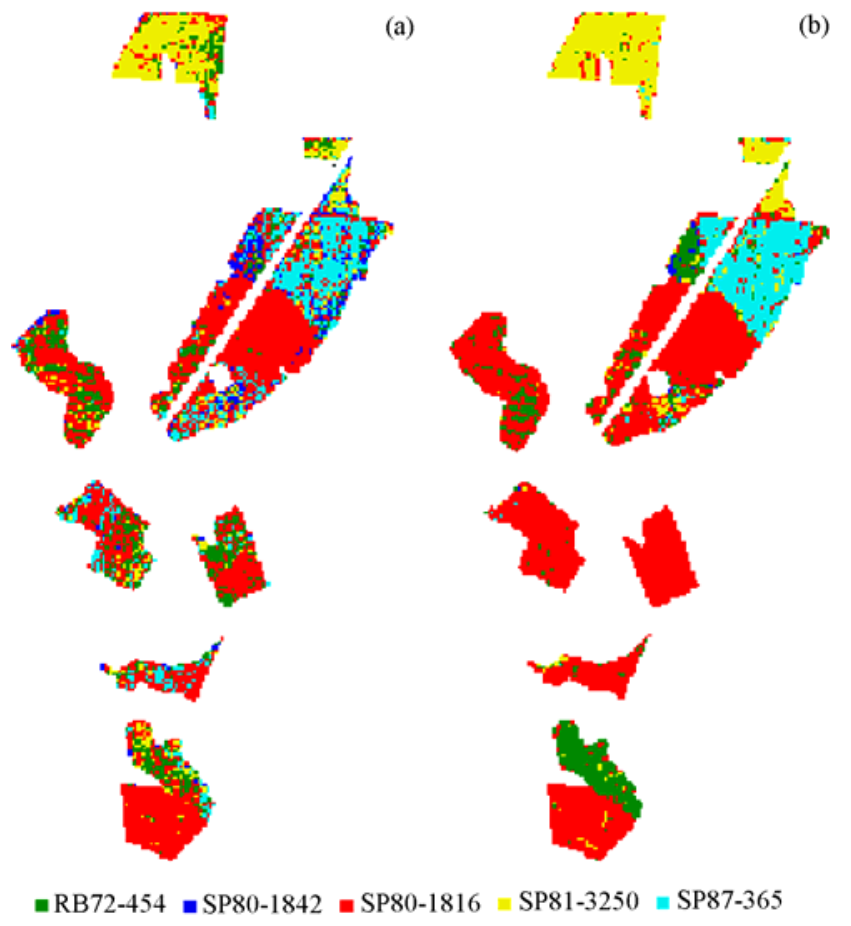

FIGURA 5. Ampliação de algumas áreas de cana-de-açúcar classificadas a partir das imagens das seis bandas refletivas do sensor $\operatorname{ETM}^{+}$(a), e das primeiras oito CPs do sensor Hyperion (b).

Nesse contexto, conforme ressaltado em GALVÃO et al. (2005), as diferenças relacionadas com diferentes estruturas de dosséis desempenham significativo papel na discriminação de variedades, considerando os dados hiperespectrais em relação aos dados multiespectrais. Além disso, os dados hiperespectrais apresentam também a importante capacidade de mostrar bandas de absorção como as da água foliar (posicionadas em $983 \mathrm{~nm}$ e em $1.205 \mathrm{~nm}$ ) e da lignina-celulose (posicionadas em $2.103 \mathrm{~nm}$ e $2.304 \mathrm{~nm}$ ), que se mostram úteis para diferenciar cultivares de canade-açúcar e, provavelmente, de outras culturas agrícolas também.

\section{CONCLUSÕES}

Em virtude da maior quantidade de informação espectral fornecida pelo sensor Hyperion, a acurácia de classificação para a imagem desse sensor foi superior à do sensor $\mathrm{ETM}^{+}$. Isso foi verificado tanto para a diferenciação das classes de uso e cobertura do solo (em que predominam diferenças espectrais marcantes entre elas) quanto para as classes de variedades de cana-de-açúcar (em que os grupos espectrais são muito semelhantes). Apesar disso, a qualidade da classificação de uso e cobertura do solo obtida a partir da imagem do sensor $\mathrm{ETM}^{+}$foi semelhante à obtida a partir do sensor Hyperion, sugerindo a suficiência de informação espectral dos dados $\mathrm{ETM}^{+}$para esse tipo de classificação; já para diferenças sutis entre alvos (diferenciação de variedades de cana-deaçúcar), o melhor resultado de classificação ocorreu a partir da imagem hiperespectral, demonstrando a importância da mesma na discriminação de alvos agrícolas que apresentam diferenças espectrais sutis.

\section{REFERÊNCIAS}

APAN, A.; HELD, A.; PHINN, S.; MARKLEY, J. Detecting sugarcane 'Orange Rust' disease using EO-1 Hyperion Hyperspectral Imagery. International Journal of Remote Sensing, London, v.25, n.2, p.489-98, 2004.

CONGALTON, R.G.; GREEN, K. Assessing the accuracy of remotely sensed data: principles and practices. Boca Raton: Lewis Publischers, 1999. 137 p. 
GALVÃO, L.S.; FORMAGGIO, A.R.; TISOT, D.A. Discrimination of sugarcane varieties in southeastern Brazil with EO-1 Hyperion data. Remote Sensing of Environment, New York, v.94, n.4, p.523-34, 2005.

GOODENOUGH, D.G.; DYK, A.; NIEMANN, O.; PEARLMAN, J.S.; CHEN, H.; HAN, T.; MURDOCH, M.; WEST, C. Processing Hyperion and ALI for forest classification. IEEE Transactions on Geoscience and Remote Sensing, New York, v.41, n.2, p.1321-31, 2003.

GÜRTLER, S.; EPIPHANIO, J.C.N.; LUIZ, A.J.B.; FORMAGGIO, A.R. Planilha eletrônica para o cálculo da reflectância em imagens TM e ETM+ Landsat. Revista Brasileira de Cartografia, Presidente Prudente, v.57, n.2, p.162-7, 2005.

HUDSON, W.D.; RAMM, C.W. Correct formulation of the kappa coefficient of agreement. Photogrammetric Engineering \& Remote Sensing, Falls Church, v.53, n.4, p.421-22, 1987.

IMSPEC. ACORNTM User's Guide. Boulder: Analytical Imaging and Geophysics, 2001. 64 p.

NASA. USGS EO-1. Disponível em: <http://eo1.usgs.gov/hyperion.php>. Acesso em: 7 mar. 2007.

PEARLMAN, J.S.; BARRY, P.S.; SEGAL, C.; SHEPANSKI, J.; BEISO, D.; CARMAN, S. Hyperion, a space-based imaging spectrometer. IEEE Transactions on Geoscience and Remote Sensing, New York, v.41, n.6, p.1160-73, 2003.

RICHARDS, J.A.; JIA, X. Remote sensing digital image analysis: an introduction. $3^{\text {rd }}$ ed. Berlin: Springer, 1999. $363 \mathrm{p}$.

SULSOFT. Guia ENVI. Disponível em:<http://intranet.sulsoft.com.br/site/guia_envi/f/index.html>. Acesso em: 9 mar. 2007.

SWAIN, P.H.; DAVIS, S.M. Remote sensing: the quantitative approach. New York: McGraw-Hill, 1978. $396 \mathrm{p}$.

THENKABAIL, P.S.; ENCLONA, E.A.; ASHTON, M.S.; LEGG, C.; DE DIEU, M. J. Hyperion, IKONOS, ALI and ETM plus sensors in the study of African rainforests. Remote Sensing of Environment, New York, v.90, n.1, p.23-43, 2004. 